\title{
Composição do óleo essencial de quatro espécies do gênero Plectranthus
}

\author{
BANDEIRA, J.M.*; BARBOSA, F.F.; BARBOSA, L.M.P.; RODRIGUES, I.C.S.; BACARIN, M.A.; PETERS, J.A.; \\ BRAGA, E.J.B. \\ Universidade Federal de Pelotas, Campus Capão do Leão, CEP: 96.100-900, Pelotas-Brasil *bandeira.j@gmail.com
}

\begin{abstract}
RESUMO: O gênero Plectranthus é considerado um dos mais ricos em óleos essenciais dentro da família Lamiaceae, compreendendo muitas espécies com propriedades medicinais. Algumas destas são conhecidas popularmente como boldo, as quais possuem semelhanças taxonômicas e diversas sinonímias, possuindo ações anti-dispépticas, analgésicas e digestivas. O objetivo deste estudo foi avaliar quantitativa e qualitativamente os óleos essenciais presentes nas folhas das espécies $P$. amboinicus, $P$. barbatus, $P$. grandis e $P$. neochilus. A extração do óleo foi realizada por hidrodestilação, utilizando pentano como solvente extrator, repetida por três vezes para cada uma das espécies. A análise dos componentes dos óleos essenciais das quatro espécies de Plectranthus, através da CG/EM, permitiu identificar 14 componentes químicos, sendo a maioria sesquiterpenos. O trans-cariofileno se apresentou em elevada concentração nos óleos estudados. Alguns componentes químicos demonstraram ser específicos para cada espécie e outros apresentaram ocorrência comum a todas as quatro, possibilitando a diferenciação das mesmas em dois grupos, um formado por $P$. amboinicus e $P$. neochilus e o outro por $P$. grandis e $P$. barbatus. Conclui-se que as quatro espécies de boldo apresentam diferenças significativas quanto ao teor e à constituição química do óleo essencial.
\end{abstract}

Palavras-chave: plantas medicinais, boldo, hidrodestilação, CG-MS, GC-FID

ABSTRACT: Essential oil composition of four Plectranthus species. Plectranthus has been considered one of the richest genera in essential oils within the Lamiaceae family, which includes several species with medicinal properties. Some of them are commonly known as boldo and present taxonomic similarities and several synonymies, with antidyspeptic, analgesic and digestive actions. The aim of this study was to evaluate quantitatively and qualitatively the essential oils from $P$. amboinicus, $P$. barbatus, $P$. grandis and $P$. neochilus leaves. The oil was extracted by hydrodistillation using pentane as extracting solvent and was repeated three times for each species. The analysis of essential oil components by GC/MS in the four Plectranthus species identified 14 chemical components, mostly sesquiterpenes. High concentration of trans-caryophyllene was found in the studied oils. Some chemical components were specific for each species and other components had common occurrence in all four species, allowing their differentiation into two groups, one composed of $P$. amboinicus and $P$. neochilus and another one by $P$. grandis and $P$. barbatus. In conclusion, the four boldo species had significant differences as to essential oil yield and chemical composition.

Key words: medicinal plants, boldo, hydrodistillation, GC-MS, GC-FID

\section{INTRODUÇÃO}

Os produtos oriundos de plantas medicinais ocupam um espaço cada vez maior na terapêutica, sobressaindo-se pela eficácia e, principalmente, pelo menor número de contra indicações e de efeitos colaterais, quando comparados aos medicamentos sintéticos (Mantel et al., 1994; Lorenzi \& Matos, 2002;
Montanari Júnior, 2003).

Dados da Organização Mundial de Saúde (OMS) demonstram que cerca de $80 \%$ da população mundial faz uso de algum tipo de erva na busca de alívio de alguma sintomatologia dolorosa ou desagradável. Desse total, pelo menos 30\% ocorreu

Recebido para publicação em 28/04/2009

Aceito para publicação em 19/10/2010

Rev. Bras. PI. Med., Botucatu, v.13, n.2, p.157-164, 2011. 
por indicação médica (Silva \& Casali, 2000). AABIFITO (2007) estima que $82 \%$ da população brasileira utilizam no tratamento de doenças, produtos à base de plantas medicinais.

No Brasil, segundo projeções do Instituto Brasileiro de Plantas Medicinais (IBPM), o mercado de medicamentos fitoterápicos movimenta de 400 milhões a 500 milhões de dólares por ano (Botsaris, 2009). Segundo o mesmo autor, no mundo, estimase que o gasto com plantas medicinais chegue à cifra de US\$27 bilhões (em torno de 7\% do mercado mundial de medicamentos). Entretanto, enquanto 0 mercado farmacêutico cresce de 3 a $4 \%$ ao ano no mundo, o fitoterápico sobe de 6 a $7 \%$.

De acordo com Sacramento (2009), no Brasil, $63 \%$ dos medicamentos disponíveis são consumidos por apenas $20 \%$ da população, o restante possui como única fonte terapêutica o uso dos recursos naturais. No entanto, até o momento, ainda não se conhece quase nada sobre a composição química de $99,6 \%$ das plantas da flora nacional, estimadas entre 40 mil a 55 mil espécies (Schultes, 1991). Entre essas plantas, podem-se destacar as pertencentes à família Lamiaceae. Além disso, grande quantidade de compostos secundários isolados de plantas medicinais, com estrutura química já determinada, ainda não foi estudada quanto às atividades biológicas (Stangarlin et al., 1999).

O gênero Plectranthus (L'Her.), entre os gêneros pertencentes à família Lamiaceae, é considerado um dos mais ricos em óleos essenciais, tendo como principais constituintes os mono e sesquiterpenos (Abdel-Mogib et al., 2002). Este gênero compreende muitas plantas de interesse medicinal e econômico, entretanto a composição química é pouco conhecida. Das 300 espécies do gênero Plectranthus já identificadas, 62 são mencionadas por possuírem propriedades medicinais, alimentícias, flavorizantes, antissépticas, repelentes e por serem utilizados como pastagens e como plantas ornamentais (Lukhoba et al., 2006). Várias espécies deste gênero são referidas como boldo, e utilizadas como plantas medicinais devido as propriedades anti-dispépticas, analgésicas e estimulantes da digestão (Lorenzi \& Matos, 2002). Muitas destas propriedades estão atribuídas à presença dos óleos essenciais, que são misturas complexas podendo conter 100 ou mais compostos orgânicos. Os constituintes podem pertencer às mais diversas classes de compostos, porém os terpenos e os fenilpropenos são as classes de compostos comumente encontradas (Hay \& Svodoba, 1993).

Dentre as espécies do gênero Plectranthus, quatro (Plectranthus grandis, Plectranthus barbatus, Plectranthus neochilus e Plectranthus amboinicus), conhecidas popularmente como boldo, se destacam na medicina popular por possuírem propriedades medicinais semelhantes. O P. barbatus Andrews e o P. grandis (Cramer) R.H. Willemse, conhecidos como falso-boldo e boldo-grande, respectivamente, são muito semelhantes morfologicamente e, por isso, são bastante confundidos, sendo ambos utilizados para os mesmos fins na medicina popular (Lorenzi \& Matos, 2002).

A espécie $P$. barbatus pode ser usada como tônico, digestivo, hipossecretor gástrico, para afecções do fígado e ressaca alcoólica (Martins et al., 2002). Possui também atividades analgésicas, antiinflamatórias, coleréticas, colagogas, antifúngica, anti-séptica e, de acordo com Bhakuni et al. (1970), apresenta também função antiviral, antitumoral, antibacteriana e ainda, ação antioxidante (Tamarisio et al., 1998) e atividades citotóxicas (Costa \& Nascimento, 2003).

O P. neochilus (Schlechtre), vulgarmente conhecido como boldo-gambá, é uma planta herbácea muito aromática, com uso semelhante ao de $P$. barbatus (Lorenzi \& Matos, 2002). De acordo com Lukhoba et al. (2006), o P. amboinicus (Lour.) Spreng. também possui propriedades medicinais semelhantes às das espécies anteriormente mencionadas.

Por causa das semelhanças taxonômicas, diversas nomenclaturas têm sido utilizadas para a mesma espécie do gênero Plectranthus, tornando difícil a coleta de informações sobre a utilização etnobotânica deste gênero. Também é importante salientar que as espécies de Plectranthus comumente utilizadas para fins medicinais possuem grande número de sinonímias (Lukhoba et al., 2006). Em razão disso, estudos em quimiotaxonomia que consistem na investigação de compostos químicos que ocorrem em plantas são importantes para determinar os compostos predominantes em cada espécie (Figueiredo-Ribeiro \& Almeida, 1986; Haslam, 1996).

Diante do exposto, o objetivo do presente trabalho foi contribuir no estudo do gênero Plectranthus, por meio da avaliação quantitativa e qualitativa dos óleos essenciais presentes nas folhas das espécies $P$. amboinicus, $P$. barbatus, $P$. grandis e $P$. neochilus.

\section{MATERIAL E MÉTODO}

\section{Material Vegetal}

Foram utilizadas plantas das quatro espécies do gênero Plectranthus ( $P$. amboinicus, $P$. barbatus, $P$. grandis e $P$. neochilus), conhecidas popularmente como boldo, as quais foram propagadas por estaquia, em vasos com $30 \mathrm{~cm}$ de diâmetro, contendo como substrato uma mistura de solo + Plantmax ${ }^{\circledR} 1: 1(\mathrm{v} / \mathrm{v})$. As plantas foram cultivadas em casa de vegetação, com umidade relativa em torno de $70 \%$ e temperatura entre 25 e $30^{\circ} \mathrm{C}$, por aproximadamente três meses (outubro a dezembro de 2007). 
Estas espécies, da família Lamiaceae, foram coletadas e devidamente identificadas pela bióloga Juliana de Magalhães Bandeira sendo um exemplar de cada espécie depositado no herbário PEL do Departamento de Botânica, da Universidade Federal de Pelotas, sob os números 24.586 ( $P$. grandis), 24.587 (P. barbatus), 24.585 ( $P$. neochilus) e 24.584 (P. amboinicus).

\section{Colheita}

A colheita dos ramos foi realizada, entre 8 e 9 horas da manhã, em quantidade suficiente para as análises que seriam realizadas durante o dia. Para isso foram selecionados os ramos mais jovens e sadios e realizado o desfolhamento, sendo as folhas sadias armazenadas em B.O.D. até o momento das análises.

\section{Determinação do teor de água}

A determinação do teor de água foi realizada pelo método gravimétrico, através da metodologia adaptada do Asae Standards (ASAE, 2000), onde amostras em triplicata, de $10 \mathrm{~g}$ de folhas recém colhidas, foram secas em estufa, com temperatura entre 65 e $70^{\circ} \mathrm{C}$, até obtenção de massa constante, o que ocorreu em torno de 72 horas. Através da diferença entre a massa fresca e a seca calculou-se a quantidade de água, que foi expressa em porcentagem de base úmida (\% b.u.). De acordo com Pimentel et al. (2006), a determinação do teor de umidade da matéria-prima é de fundamental importância para comparar os resultados da quantificação do óleo essencial entre diferentes tratamentos ou mesmo espécies, utilizando como padrão a porcentagem de base seca (\% b.s.).

\section{Extração e quantificação dos óleos essenciais}

A extração foi realizada por hidrodestilação, utilizando o aparelho Clevenger adaptado a um balão de fundo redondo de $1 \mathrm{~L}$, onde foram colocadas 100 $\mathrm{g}$ de folhas frescas submersas em $500 \mathrm{~mL}$ de água destilada, com aquecimento mantido na temperatura mínima necessária à ebulição. Com o objetivo de facilitar a extração, as folhas foram cortadas transversalmente a cada $1 \mathrm{~cm}$. O tempo de extração foi de cinco horas, contados a partir do momento da ebulição, o qual foi determinado através de testes preliminares. Durante este procedimento foram coletadas alíquotas de $10 \mathrm{~mL}$ a cada hora, para não ocorrer o refluxo do óleo. Na última coleta, após as cinco horas, a coluna de extração do Clevenger foi lavada com solvente (pentano) para recolher possíveis resíduos de amostra. Foram realizadas três extrações de cada uma das espécies.

O material coletado durante a extração foi colocado em funil de separação de $500 \mathrm{~mL}$, onde o óleo essencial foi separado da fase aquosa utilizando pentano $(10 \mathrm{~mL})$ como solvente extrator. Este processo foi repetido por três vezes. A fração orgânica (óleo+solvente) proveniente do funil de separação foi coletada em erlenmeyer de $100 \mathrm{~mL}$ e em seguida tratada com sulfato de magnésio anidro $\left(\mathrm{MgSO}_{4}\right)$ em excesso, para a retirada da água remanescente.

A seguir, a solução foi filtrada e, em seguida, concentrada em evaporador rotativo a $40^{\circ} \mathrm{C}$, até atingir quantidade suficiente para possibilitar a transferência para frasco de $10 \mathrm{~mL}$, sendo este colocado em banho-maria à temperatura de $40^{\circ} \mathrm{C}$, até atingir massa constante.

A quantificação do óleo essencial foi realizada através de pesagem em balança analítica, com precisão de $0,0001 \mathrm{~g}$, sendo o resultado expresso em porcentagem em base seca.

Após a quantificação, os recipientes com os óleos essenciais foram vedados com parafilme, envoltos em papel alumínio e armazenados em ultrafreezer $\left(-80^{\circ} \mathrm{C}\right)$, para posteriormente serem analisados por cromatografia gasosa.

\section{Análises cromatográficas}

As análises cromatográficas foram realizadas no Laboratório de Análise e Síntese de Agroquímicos, localizado no Departamento de Química da Universidade Federal de Viçosa, sendo as análises conduzidas de acordo com Barbosa et al. (2006).

A identificação dos constituintes químicos foi realizada empregando-se cromatografia em fase gasosa associada à espectrometria de massas (CGMS). A metodologia utilizada para identificação dos componentes foi a descrita por Adams (2007), sendo empregada coluna DB-5HT (J \& W Scientific $\left.{ }^{\circledR}\right)$, com $30 \mathrm{~m}$ de comprimento, $0,32 \mathrm{~mm}$ de diâmetro interno e $10 \mu \mathrm{m}$ de espessura do filme interno, utilizando hélio como gás carreador. $O$ cromatógrafo gasoso teve as seguintes condições de operação: pressão interna da coluna de $56,7 \mathrm{kPa}$, razão de split de 1:20, fluxo de gás na coluna de $1,0 \mathrm{~mL} \mathrm{~min}^{-1}$, velocidade linear do gás de $36,5 \mathrm{~cm} \mathrm{~s}^{-1}$, temperatura no injetor de $220^{\circ} \mathrm{C}$, temperatura no detector de $240^{\circ} \mathrm{C}$. A temperatura da coluna foi programada para iniciar em $60^{\circ} \mathrm{C}$ e permanecer por $1 \mathrm{~min}$, após, um incremento de $3^{\circ} \mathrm{C}$ por minuto até atingir $240^{\circ} \mathrm{C}$, e mantida por mais 30 min, totalizando 91 min. O espectrômetro de massas foi programado para realizar leituras numa faixa de 29 a $400 \mathrm{~m} / \mathrm{z}$, em intervalos de $0,5 \mathrm{~s}$, com energia de ionização de $70 \mathrm{eV}$ e temperatura do detector de $240^{\circ} \mathrm{C}$. O volume de amostra injetado foi de $1 \mu \mathrm{L}$, em concentração de 10.000 ppm, utilizando como solvente o hexano. Para a identificação dos componentes, além dos espectros de massa sugeridos pela biblioteca presente no aparelho, foram calculados os índices de retenção relativos (índices de Kovats).

Para o cálculo dos índices de Kovats, foi injetada no cromatógrafo uma mistura de hidrocarbonetos (C8 a C24), sendo que para cada constituinte do óleo essencial foram utilizados os 
tempos de retenção do próprio constituinte e dos hidrocarbonetos, usando a equação descrita por Collins et al. (2006).

A quantificação dos componentes do óleo essencial foi realizada empregando-se cromatografia em fase gasosa associada à deteç̧ão por ionização de chamas (GC-FID), utilizando-se o cromatógrafo gasoso modelo Shimadzu GC-17A, acoplado ao detector por ionização de chamas, sendo utilizadas colunas SPB-5 (Supelco ${ }^{\circledR}$ ), com 30 m de comprimento, $0,25 \mathrm{~mm}$ de diâmetro interno e $0,25 \mu \mathrm{m}$ de espessura do filme interno, utilizando nitrogênio como gás carreador. As condições de operação do equipamento foram pressão interna da coluna de $100 \mathrm{kPa}$, razão de split de 1:10, fluxo de gás na coluna de 1,33 $\mathrm{mL} \mathrm{min}^{-1}$, velocidade linear do gás $31,80 \mathrm{~cm} \mathrm{~s}^{-1}$, temperatura no injetor e detector de $250^{\circ} \mathrm{C}$. A temperatura da coluna foi programada para iniciar em $60^{\circ} \mathrm{C}$ e permanecer por 1 min, após, um incremento de $3^{\circ} \mathrm{C}$ por minuto até atingir $240^{\circ} \mathrm{C}$, totalizando o tempo de $61 \mathrm{~min}$. O volume de amostra injetada foi de $1 \mu \mathrm{L}$, em concentração de 10.000 ppm e utilizando como solvente o hexano. Para quantificar cada constituinte do óleo essencial foram realizadas integrações das áreas geradas e calculadas pelo programa presente no próprio computador conectado ao GC-FID, sendo os resultados expressos em percentual proporcional de área. Aidentificação de cada componente foi feita comparando-se os tempos de retenção, os índices de Kovats e os cromatogramas gerados no GC-MS e no GC-FID.

\section{Delineamento experimental e análises estatísticas}

O delineamento experimental foi inteiramente casualizado com três repetições por espécie, sendo cada uma representada por três plantas. Para os cálculos estatísticos, foram realizadas análises de variância, de desvios padrão e testes de comparação múltipla de médias utilizando o software Winstat 2.0 (Machado \& Conceição, 2003).

\section{RESULTADO E DISCUSSÃO}

\section{Teor de água}

A partir das análises dos resultados, verificouse que houve diferença estatística em relação ao teor de água nas folhas das espécies analisadas, sendo que a maior média foi apresentada para o $P$. neochilus, com $93,12 \%$ b.u., não diferindo estatisticamente do $P$. amboinicus. Jáa menor média ( $87,16 \%$ b.u.) foi observada na $P$. barbatus, não diferindo estatisticamente da $P$. grandisque apresentou média de $88,23 \%$ b.u. (Figura 1).

Os resultados se apresentaram de acordo com o esperado, já que as espécies $P$. neochilus e $P$. amboinicus se assemelham morfologicamente, pois ambas possuem folhas pequenas e suculentas, com aproximadamente $6 \mathrm{~cm}$ de comprimento. Já as outras duas espécies ( $P$. barbatus e $P$. grandis) são bastante confundidas entre si, possuindo folhas maiores (chegando a $12 \mathrm{~cm}$ de comprimento) e mais largas (com aproximadamente $10 \mathrm{~cm}$ de largura), além de serem menos espessas que as primeiras.

\section{Quantificação do óleo essencial}

Observou-se diferença significativa em relação à quantificação do óleo essencial para as quatro espécies estudadas (Figura 2). A espécie $P$. amboinicus foi a que apresentou o maior teor de óleo essencial, em média, $0,43 \%$ b.s., não diferindo estatisticamente da $P$. neochilus que apresentou em média $0,32 \%$. Já as espécies $P$. barbatus e $P$. grandis foram as que apresentaram os menores valores, em média, $0,10 \%$ b.s. e $0,09 \%$ b.s., respectivamente.

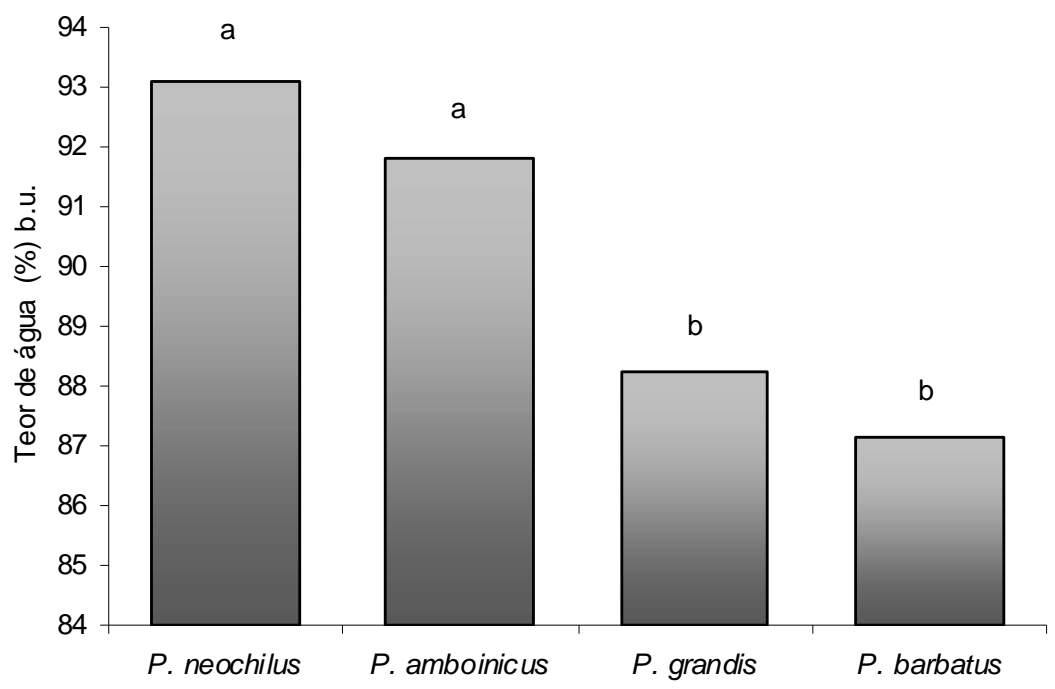

FIGURA 1. Teor de água das folhas de quatro espécies de Plectranthus, obtida pela diferença de massa fresca e seca. Letras distintas representam diferença estatística ao nível de 5\% de probabilidade pelo teste de Tukey. 


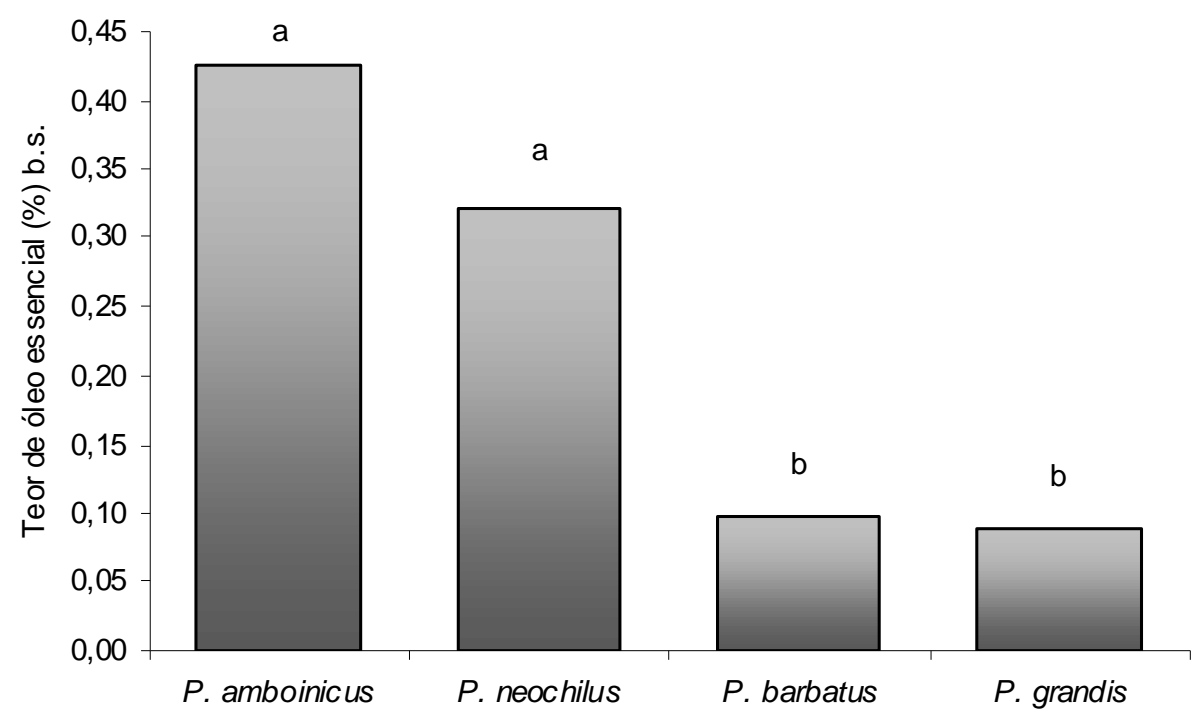

FIGURA 2. Teor de óleo essencial extraído de folhas frescas de quatro espécies de Plectranthus. Letras distintas representam diferença estatística ao nível de $5 \%$ de probabilidade pelo teste de Tukey.

A hidrodestilação das folhas frescas das espécies em estudo forneceu óleo de cor amarelada, com um odor forte característico, mas com rendimento muito baixo em relação ao material fresco, provavelmente em função do elevado teor de umidade nas folhas, dados também observados por Bocardi (2008) através de estudos com $P$. neochilus que apresentou $0,024 \%$ b.s. de rendimento do óleo com um teor de água de 93,7\%.

O comportamento diferenciado entre as espécies é intrínseco das características morfológicas. Foi possível observar que a concentração de óleos essenciais por grama de massa seca é menor para $P$. grandis e $P$. barbatus, e maior para $P$. neochilus e $P$. amboinicus, demonstrando a existência de maior proximidade e similaridade entre estas espécies, estes resultados estão de acordo com as análises moleculares, dessas mesmas espécies, obtidas por Bandeira et al. (2010).

De acordo com Abdel-Mogib (2002), a constituição química das espécies do gênero Plectranthus é pouco conhecida, sendo que estas são ricas em óleos essenciais, possuindo na constituição teores abaixo de $0,5 \%$ de óleo essencial em base seca, concordando com os baixos valores encontrados no presente trabalho.

\section{Identificação e quantificação dos componentes dos óleos essenciais}

Os resultados da quantificação e identificação dos componentes dos óleos essenciais das quatro espécies de Plectranthus, através da análise por $C G / E M$, permitiram identificar um total de 14 componentes químicos, distribuídos nas 4 espécies (Tabela 1). Conforme observado, no presente trabalho, a maioria dos compostos identificados são sesquiterpenos, corroborando dados encontrados por Abdel-Mogib (2002), que afirma que os maiores constituintes dos óleos essenciais deste gênero são os mono e sesquiterpenos. Este mesmo autor salienta que cerca de 140 diterpenóides já foram identificados, de glândulas foliares, para espécies de Plectranthus.

É possível observar que o componente que se apresenta em elevada concentração nos óleos estudados é o trans-cariofileno. Este composto representa cerca de $25,5 \%$ do óleo obtido de $P$. neochilus e $P$. amboinicus, sendo encontrado em menor escala em $P$. barbatus e $P$. grandis. Este composto também foi encontrado como constituinte majoritário em $P$. neochilus no estudo realizado por Bocardi (2008), porém em concentração menor $(19,96 \%)$.

O cariofileno é um sesquiterpeno sintetizado pelas plantas na rota metabólica dos terpenos, sendo também importante constituinte do óleo essencial de outras espécies medicinais, tais como, Syzygium aromaticum L. (Barbosa, 1998), Copaifera langsdorfii Desf. (Feibert \& Langenheim, 1988) e Xylopia brasiliensis Speng. (Santos et al., 2004). Craveiro et al. (1981) ressaltaram que em Annona squamosa L. este composto é encontrado no óleo essencial, com um rendimento de $0,4 \%$ e um teor de $63 \%$. De acordo com Ascensão et al. (1998), o $\beta$-cariofileno é um dos principais constituintes químicos encontrados em diferentes espécies do gênero Plectranthus ( $P$. rugosus, $P$. fruticosus, $P$. coleoides, $P$. tenuiflorus, $P$. incanus e $P$. defoliatus).

Os óleos essenciais apresentam atividades medicinais, podendo ser utilizados, de acordo com as características, para diversas finalidades (Barbosa, 1998). O óleo essencial rico em cariofileno, segundo

Rev. Bras. PI. Med., Botucatu, v.13, n.2, p.157-164, 2011. 
TABELA 1. Composição química majoritária do óleo essencial de folhas de quatro espécies do gênero Plectranthus (valores expressos em percentual proporcional da área).

\begin{tabular}{ccccccc}
\hline No & Composição do óleo & $\mathbf{I K}^{2}$ & $\begin{array}{c}\text { Plectranthus } \\
\text { grandis }\end{array}$ & $\begin{array}{c}\text { Plectranthus } \\
\text { amboinicus }\end{array}$ & $\begin{array}{c}\text { Plectranthus } \\
\text { neochilus }\end{array}$ & $\begin{array}{c}\text { Plectranthus } \\
\text { barbatus }\end{array}$ \\
\hline 1 & $\alpha$-Cubebeno & 1351 & - & $2,57 \pm 0,08$ & $0,68 \pm 0,05$ & - \\
2 & $\alpha$-Copaeno & 1376 & $14,89 \pm 0,30$ & $3,13 \pm 0,11$ & $1,17 \pm 0,04$ & $5,42 \pm 0,06$ \\
3 & $\beta$-Bourboneno & 1384 & $3,88 \pm 0,29$ & $6,30 \pm 0,28$ & $1,99 \pm 0,06$ & $4,78 \pm 1,54$ \\
4 & trans-Cariofileno & 1418 & $12,94 \pm 0,23$ & $25,53 \pm 0,96$ & $25,68 \pm 0,66$ & $21,02 \pm 1,64$ \\
5 & $\alpha$-humuleno & 1454 & - & $2,11 \pm 0,02$ & $1,25 \pm 0,04$ & $1,42 \pm 0,03$ \\
6 & Germacreno-D & 1480 & $7,56 \pm 1,15$ & $3,98 \pm 0,01$ & $4,18 \pm 0,12$ & $15,52 \pm 0,06$ \\
7 & Valenceno & 1489 & - & - & - & $22,85 \pm 0,09$ \\
8 & $\alpha$-Zingibereno & 1495 & $4,12 \pm 0,16$ & - & - & - \\
9 & $\alpha$-Chamigreno & 1500 & - & - & $8,81 \pm 0,19$ & - \\
10 & $\alpha$-Bulneseno & 1505 & - & $5,70 \pm 1,08$ & - & - \\
11 & $\beta$-Bisaboleno & 1508 & $3,04 \pm 0,11$ & - & - & $3,27 \pm 0,02$ \\
12 & Isobornil 2-metilbutirato & 1513 & $6,05 \pm 0,19$ & - & - & - \\
13 & $\Delta$-Cadineno & 1519 & $4,50 \pm 0,45$ & $4,40 \pm 0,71$ & $1,85 \pm 0,06$ & $3,13 \pm 0,02$ \\
14 & Óxido de cariofileno & 1579 & - & $9,76 \pm 0,42$ & $2,77 \pm 0,10$ & $3,86 \pm 0,06$ \\
\hline & Total identificado & & $69,58 \pm 2,88$ & $62,47 \pm 0,07$ & $48,38 \pm 1,12$ & $81,27 \pm 0,20$ \\
\hline
\end{tabular}

Médias de três extrações independentes seguidas dos desvios padrão. 2Índices de Kovats calculados.

Haslam (1996), pode ser empregado na medicina tradicional como remédio, para o tratamento de diversas moléstias orgânicas. A ocorrência de cariofileno como principal componente do óleo essencial pode estar relacionada ao uso tradicional destas espécies vegetais contra as dores estomacais (Bocardi, 2008).

Em P. grandis, o componente químico encontrado em maior concentração foi o $\alpha$-copaeno, representando $15 \%$ do óleo essencial, seguido do trans-cariofileno com aproximadamente $13 \%$. Nas demais espécies, o $\alpha$-copaeno apresentou concentração muito baixa.

O componente majoritário encontrado no óleo de $P$. barbatus foi o Valencene, representando cerca de $23 \%$ da composição, seguido do trans-cariofileno com $21 \%$ e do germacreno-D com $15 \%$. Foi observado também que o valenceno não estava presente em nenhuma das outras espécies estudadas.

De acordo com Matos (2000), a análise dos fitoquímicos de $P$. barbatus registrou a presença de
$0,1-0,3 \%$ do óleo essencial rico em guaieno e fenchona, que são responsáveis pelo aroma característico, e compostos fixos como os terpenos e barabatusina além de outros compostos, acreditando que estes compostos sejam responsáveis pela proteção ao ataque de patógenos. Segundo Chalchat et al. (1996), vários terpenos labdados têm sido isolados de exemplares de $P$. barbatus, sendo que nas análises 25 dos 91 constituintes foram identificados na análise do óleo de baixo rendimento $(0,05 \%)$, tendo como principais constituintes o fencil acetato, $\alpha$-copaeno, aromadendreno, borneol, $\alpha^{2}-$ candineno, trans-cariofileno, ledol, T-cadinol e torreiol. No óleo essencial das folhas de $P$. barbatus foram encontrados como constituintes principais $\alpha$-pineno $(22,2 \%)$, mirceno (12,3\%), (Z)- $\beta$-ocimeno $(6,5 \%), \beta$ cariofileno $(8,0 \%)$ e humuleno $(10,0 \%)$ (Kerntopf et al., 2002).

Outros componentes também são específicos para cada espécie tais como o $\alpha$ zingiberene e Isobornil 2-metilbutirato, presentes 
apenas em $P$. grandis, assim como $\alpha$-bulneseno e $\alpha$ chamigreno exclusivamente de $P$. amboinicus e $P$. neochilus, respectivamente.

Pode-se observar também a ocorrência dos componentes $\triangle$-cadineno, $\alpha$-copaeno, $\beta$-bourboneno, trans-cariofileno e $\alpha$-humuleno presentes nas quatro espécies estudadas. Entretanto cada uma delas apresentou composição química diferenciada.

A abundância de substâncias voláteis provém da rota metabólica dos isoprenóides, que culmina na formação do sesquiterpeno cujo precursor é o farnesildifostato. Esse precursor, com a ação de monoxigenases (enzimas ativadas pelo oxigênio), forma trans-cariofileno, germacreno- $D$, $\alpha$-humuleno, entre outros e compõem a maioria dos compostos voláteis, os quais segundo a literatura apresentam atividades antibacterianas, fungicidas e inseticidas (Almeida et al., 2005).

Através da análise dos componentes químicos dos óleos essenciais destas espécies, é possível verificar a formação de dois grupos. Um formado por $P$. amboinicus e $P$. neochilus e outro por $P$. grandis e $P$. barbatus. $\mathrm{O} \alpha$-cubebeno está presente apenas em $P$. amboinicus e $P$. neochilus enquanto que o $\beta$-bisaboleno somente é encontrado em $P$. grandis e $P$. barbatus, demonstrando a existência de maior proximidade e similaridade entre estas espécies, corroborando com dados obtidos através de análises moleculares, por Bandeira et al. (2010), onde foi observada maior similaridade genética $(80 \%)$ entre as espécies $P$. neochilus e $P$. amboinicus, seguido por $P$. grandis e $P$. barbatus (77\%).

Com o estudo pode-se concluir que as quatro espécies de boldo ( $P$. amboinicus, $P$. barbatus, $P$. grandis e $P$. neochilus) apresentam diferenças significativas quanto ao teor e à constituição química do óleo essencial. Pode-se observar que o transcariofileno é o componente que se apresenta em elevada concentração nos óleos das plantas estudadas, sendo o componente majoritário do óleo de $P$. amboinicus e $P$. neochilus. Nas espécies $P$. grandis e $P$. barbatus os componentes químicos que se apresentam em maior quantidade são $\alpha$-copaeno e valenceno, respectivamente.

\section{AGRADECIMENTO}

Ao professor PhD Luiz Cláudio de Almeida Barbosa e ao Técnico de Laboratório Químico José Luis Pereira, pertencentes ao Laboratório de Análise e Síntese de Agroquímicos da Universidade Federal de Viçosa.

À Coordenação de Apoio de Aperfeiçoamento de Pessoal de Nível Superior - CAPES e Conselho Nacional de Desenvolvimento Científico e Tecnológico CNPq, pela concessão de bolsas de estudo.

\section{REFERÊNCIA}

ABDEL-MOGIB, M.; ALBAR, H.A.; BATTERJEE, S.M. Chemistry of the Genus Plectranthus. Molecules, v.7, p.271-301, 2002.

ADAMS, R.P. Identification of essential oils components by gas chromatography mass spectroscopy. 4.ed. Illinois: Allured Publishing Corporation, Carol Stream, 2007. 804p.

ALMEIDA, L.F.R.; DELACHIAVE, M.E.A.; MARQUES, M.O.M. Composição do óleo essencial de rubim (Leonurus sibiricus L. - Lamiaceae). Revista Brasileira de Plantas Medicinais, v.8, n.1, p.35-8, 2005.

AMERICAN SOCIETY OF AGRICULTURAL ENGINEERS.

Asae Standards: standards engineering practices data. Saint Joseph: American Society of Agricultural Engineers, 2000. 565p.

ASCENSÃO, L. et al. Plectranthus madagascariensis: morphology of the glandular trichomes, essential oil composition, and its biological activity. International Journal of Plant Sciences, v.159, p.31-8, 1998.

ASSOCIAÇÃOBRASILEIRADA INDÚSTRIA FITOTERÁPICA - ABIFITO. Disponível em: <www. abifito.com.br>. Acesso em: 18 out. 2007.

BANDEIRA, J.M. et al. Genetic similarities among four species of the Plectranthus (L'Hér.) genus. Acta Scientiarum Biological Sciences, v.32, n.1, p.43-8, 2010. BARBOSA, L.C.A. Química orgânica: uma introdução para as ciências agrárias e biológicas. Viçosa: UFV, 1998. 354p.

BARBOSA, L.C.A. Introdução à química orgânica. São Paulo: Pearson Prentice-Hall, 2004. 311p.

BARBOSA, F.F. et al. Influência da temperatura do ar de secagem sobre o teor e a composição química do óleo essencial de Lippia alba (Mill.) N. E. Brown. Química Nova, v.29, n.6, p.1221-5, 2006.

BHAKUNI, D.S.; DHAWAN, B.N.; MEHROTRA, B.N. Dhar Screening of Indian plants for biological activity: part III. Indian Journal of Experimental Biology, v.9, p.91-102, 1970.

BOCARDI, J.M.B. Etnofarmacologia das plantas medicinais de céu azul e composição química do óleo essencial de Plectranthus neochilus Schltr. 2008. 101p. Dissertação (Mestrado em Química Aplicada) Universidade Estadual de Ponta Grossa, Ponta Grossa. BOTSARIS, A. Cresce interesse pela fitoterapia. Vya estelar: caminhos para o bem-estar integral. Disponível em: <http://www2.uol.com.br/vyaestelar/plantas.htm>. Acesso em: 16 mar. 2009.

CHALCHAT, J.C.; GARRY, R.; MUHAYIMANA, A. Plantas aromáticas de Ruanda. Composição química de duas Labiaceae, Plectranthus barbatus e Plectranthus sylvestris. Revista Italiana Delle Essenze, Dei Profumi e Delle Piante Officinale, v.7, p.665-74, 1996.

CRAVEIRO, A.A. et al. Óleos essenciais de plantas do nordeste. 5.ed. Fortaleza: Edições UFC, 1981. 210p.

COSTA, M.C.; NASCIMENTO, S.C. Atividade citotóxica de Plectranthus barbatus Andr. (Lamiaceae). Revista Acta Farmacêutica Bonaerense, v.22, n.2, p.155-8, 2003. COLLINS, C.H. et al. Fundamentos de cromatografia. Campinas: Editora da UNICAMP, 2006. 452p.

FEIBERT, E.B.; LANGENHEIM, J.H. Leaf resin variation in Copaifera langsdorffi: relation to irradiance and 
herbivory. Phytochemistry, v.7, n.8, p.2527-32, 1988. FIGUEIREDO-RIBEIRO, R.C.L.; ALMEIDA, V.P. Análise enzimática e quimiotaxonomia de duas variedades de Ocimum nudicaule Benth. Revista Brasileira de Botânica, v.9, n.1, p.75-80, 1986.

HASLAM, E. Natural polyphenols (vegetable tannins) as drugs and medicines: possible modes of action. Journal of Natural Products, v.59, p.205-15, 1996.

HAY, R.K.M.; SVOBODA, K.P. Botany. In: HAY, R.K.M.; WATERMAN, P.G. Volatile oil crops: their biology, biochemistry and production. Harlow: Longman Scientific \& Techinical, 1993. p.5-22.

KERNTOPF, M.R. et al. Essential oils from leaves, stems and roots of Plectranthus barbatus Andr. (Labiatae) grown in Brazil. Journal of Essential Oil Research, v.14, p.101-2, 2002.

LORENZI, H.; MATOS, F.J.A. Plantas medicinais no Brasil: nativas e exóticas. Nova Odessa: Instituto Plantarum de Estudo da Flora, 2002. 544p.

LUKHOBA, C.W.; SIMMONDS, M.S.J.; PATON, A.J. Plectranthus: A review of ethnobotanical uses. Journal of Ethnopharmacology, v.103, p.1-24, 2006.

MACHADO, A.A.; CONCEIÇÃO, A.R. Sistema de análise estatística para Windows. Winstat. Versão 2.0. UFPel, 2003.

MANTEL, S.H.; MATTHEUS, J.A.; McKEE, R.A. Princípios de biotecnologia em plantas: uma introdução à engenharia genética em plantas. Ribeirão Preto: SBG, 1994. 333p.

MARTINS, E.R. et al. Plantas medicinais. Viçosa: UFVMG, 2002. 220p.

MATOS F.J.A. Plantas medicinais. Guia de seleção de plantas usadas em fitoterapia no nordeste do Brasil. 2.ed. Fortaleza: Ed. Imprensa Universitária UFC, 2000. $344 p$.

MONTANARI JUNIOR, I. Aspectos da produção comercial de plantas medicinais nativas. Campinas: CPQBA, UNICAMP, 2003. Disponível em: <www.cpqba. unicamp.br/plmed/artigos/produção.htm>. Acesso em: 15 ago. 2007.

PIMENTEL, F.A. et al. A convenient method for the determination of moisture in aromatic plants. Química Nova, v.29, n.2, p.373-5, 2006.

SACRAMENTO, H.T. Perspectivas da fitoterapia latino americana no novo milênio. Disponível em: <http:// paginas.unisul.br/acpm/bol002.htm>. Acesso em: 16 mar. 2009.

SANTOS, B.R. et al. Aspectos da anatomia e do óleo essencial em folhas de pindaíba (Xylopia brasiliensis Spreng.). Ciência e Agrotecnologia, v.28, n.2, p.345-9, 2004.

SCHULTES, R.E. Members of Euphorbiaceae in primitive and advanced societies. Botanical Journal of the Linnean Society, v.94, n.1, p.79-95, 1991.

SILVA, F.; CASALI, V.W.D. Plantas medicinais e aromáticas: pós-colheita e óleos essenciais. Viçosa: Arte e Livros, 2000. 135p.

STANGARLIN, J.R. et al. Plantas medicinais e controle alternativo de fitopatógenos. Revista Biotecnologia Ciência \& Desenvolvimento, v.11, p.16-21, 1999.

TAMARISIO, V. et al. In vitro antioxidant activity of Coleus barbatus (Andr.) Benth (false boldo) and Peumus boldo (Molina) (Boldo do Chile): a comparative study. Revista de Farmácia e Bioquímica da Universidade de São Paulo, v.34, p.15-7, 1998. 